Mathematical Modelling and Analysis

Volume 20 Number 4, July 2015, 457-470

http://dx.doi.org/10.3846/13926292.2015.1065293

(c) Vilnius Gediminas Technical University, 2015
Publisher: Taylor\&Francis and VGTU

http://www.tandfonline.com/TMMA

ISSN: $1392-6292$

eISSN: 1648-3510

\title{
A Note on Existence Results for a Class of Three-Point Nonlinear BVPs
}

\section{Amit K. Verma and Mandeep Singh}

\author{
Department of Mathematics, BITS Pilani \\ Pilani, 333031 Rajasthan, India \\ E-mail(corresp.): amitkverma02@yahoo.co.in \\ E-mail: mandeep04may@yahoo.in
}

Received July 20, 2014; revised June 9, 2015; published online July 15, 2015

Abstract. This article deals with a computational iterative technique for the following second order three point boundary value problem

$$
\begin{aligned}
& y^{\prime \prime}(t)+f\left(t, y, y^{\prime}\right)=0, \quad 0<t<1, \\
& y(0)=0, \quad y(1)=\delta y(\eta)
\end{aligned}
$$

where $f(I \times R, R), I=[0,1], 0<\eta<1, \delta>0$. We consider simple iterative scheme and develop a monotone iterative technique. Some examples are constructed to show the accuracy of the present method. We show that our technique is quite powerful and some user friendly packages can be developed by using this technique to compute the solutions of the nonlinear three point BVPs whose close form solutions are not known.

Keywords: nonlinear boundary value problems, Green's function, three point BVP, monotone iterative technique, upper and lower solutions.

AMS Subject Classification: 34B05; 34B15; 34B10; 65L10; 47J25.

\section{Introduction}

In several real life problems, e.g., $[19,21]$, the boundary value conditions do not rely only at the end points, but also at the interior points of the interval. Such problems are known as multipoint boundary value problems. Several results are available in literature related to multipoint and nonlocal BVPs, e.g., $[1,4,5,6,8,9,10,11,12,15,16,19,20,21]$. S. Roman along with A. Štikonas $[13,14,17,18]$ established results related to construction of Green's function for nonlocal and multipoint boundary value problems.

Kiguradze and Lomtatidze [4] considered $u^{\prime \prime}(t)=f\left(t, u, u^{\prime}\right)$, where $f\left(t, u, u^{\prime}\right)=p_{0}(t)+p_{1}(t) u+p_{2}(t) u^{\prime}$, where $p_{i}(t): i=0,1,2$ are locally integrable on $] a, b[$. They considered the following three types of boundary conditions: 


$$
\begin{aligned}
& u(a+)=\alpha, \lim _{t \rightarrow b-} \frac{u^{\prime}(t)}{\sigma\left(p_{2}\right)(t)}=\beta, \\
& u(a+)=\alpha, u(b-)=\beta, \\
& u(a+)=\alpha, u(b-)=u\left(t_{0}\right)+\beta,
\end{aligned}
$$

where $-\infty<a<t_{0}<b<+\infty$ and $\sigma\left(p_{2}\right)(t)=\exp \left(\int_{(a+b) / 2}^{t} p_{2}(\tau) d \tau\right)$. They proved existence of unique solution and relaxed the condition on $p_{i}(t): i=$ $0,1,2$ such that $p_{i}$ s can be even non integrable on $[a, b]$.

Li et al. [5,6] studied the existence and uniqueness of solutions of second order 3 point boundary value problems with upper and lower solutions in reverse order. They used monotone iterative method. In a recent work Bao et al. [1] and Singh et al. $[15,16]$ prove some new results for similar 3 point boundary value problems. The functional version of these problems is considered by Kiguradze and Puza [3] and Lomtatidze and Vodstrch [7].

In this paper we present some new existence results for second order non linear 3 point boundary value problem with Dirichlet type boundary condition

$$
\begin{aligned}
& y^{\prime \prime}(t)+f\left(t, y, y^{\prime}\right)=0, \quad 0<t<1, \\
& y(0)=0, \quad y(1)=\delta y(\eta),
\end{aligned}
$$

where $f(I \times R, R), I=[0,1], 0<\eta<1, \delta>0$.

The result of this paper is an improvement over a recent result due to Bao et al. [1]. They assume two conditions $f(t, 0,0)=0$ and $y f\left(t, y, y^{\prime}\right) \geq 0$ for $y \geq 0$. Consider $-y^{\prime \prime}=h(t)+y$ which is linear but $f(t, 0,0) \neq 0$. So $f(t, 0,0)=0$ fails. Another simple example is $-y^{\prime \prime}=\sin y$. Here $y \sin y$ will change its sign for $y \geq 0$, so the condition $y f\left(t, y, y^{\prime}\right) \geq 0$ for $y \geq 0$ fails. But for both these problems the results of this paper are applicable.

Here we are looking for a simple monotone iterative scheme and propose the following

$$
\begin{aligned}
& -y_{n+1}^{\prime \prime}-\lambda y_{n+1}=f\left(t, y_{n}, y_{n}^{\prime}\right)-\lambda y_{n}, \\
& y_{n+1}(0)=0, \quad y_{n+1}(1)=\delta y_{n+1}(\eta) .
\end{aligned}
$$

We have considered " $\lambda$ " as a constant.

Cherpion et al. [2, Section 5.4] stated that (1.3)-(1.4) with constant $\lambda$ do not work. Also they [2, Remark 5.4 ] state that due to lack of uniform anti maximum principle it seems impossible to develop monotone iterative technique for reverse ordered upper and lower solution.

Remark 1. In the present work we have shown that even with constant $\lambda$ monotone sequences can be generated. Though Remark by Cherpion et al. [2, Remark 5.4 ] appears to be true for 3 point BVP also and we observe that uniform antimaximum principle does not exist.

\section{Preliminary}

Here we consider the linear 3 point BVP. We prove maximum principle and also prove existence of some differential inequalities. Consider the corresponding nonhomogeneous linear 3 point BVP 


$$
\begin{aligned}
& L y \equiv-y^{\prime \prime}(t)-\lambda y(t)=h(t), \quad 0<t<1, \\
& y(0)=0, \quad y(1)=\delta y(\eta)+b,
\end{aligned}
$$

where $h \in C(I), \& b$ any constant.

Case I: $\lambda>0$. Let us assume

$\left(H_{0}\right) 0<\lambda<\frac{\Pi^{2}}{4}, \cos \sqrt{\lambda}-\delta \cos \sqrt{\lambda} \eta \leq 0, \delta \sin \sqrt{\lambda} \eta-\sin \sqrt{\lambda}<0$.

We can easily verify that there is a range of $\lambda$, which support $\left(H_{0}\right)$ (see Figure 1).

Lemma 1. The Green's function of the 3 point $B V P L y=0, y(0)=0, y(1)=$ $\delta y(\eta)$ for $\lambda>0$, is

$$
G(t, s)=k_{1} \begin{cases}{[\sin \sqrt{\lambda}(1-s)-\delta \sin \sqrt{\lambda}(\eta-s)] \sin \sqrt{\lambda} t,} & 0 \leq t \leq s \leq \eta, \\ \sin \sqrt{\lambda} s[\sin \sqrt{\lambda}(1-t)-\delta \sin \sqrt{\lambda}(\eta-t)], & s \leq t, s \leq \eta, \\ \sin \sqrt{\lambda}(1-s) \sin \sqrt{\lambda} t, & t \leq s, \eta \leq s, \\ \delta \sin \sqrt{\lambda} \eta \sin \sqrt{\lambda}(t-s)+\sin \sqrt{\lambda} s \sin \sqrt{\lambda}(1-t), & \eta \leq s \leq t \leq 1,\end{cases}
$$

where $k_{1}=\frac{1}{\sqrt{\lambda}(\delta \sin \sqrt{\lambda} \eta-\sin \sqrt{\lambda})}$. If $\left(H_{0}\right)$ is true then $G(t, s) \leq 0$.

Proof. The Green's function for the 3 point BVP $L y=0, y(0)=0, y(1)=$ $\delta y(\eta)$ for $\lambda>0$, is defined as

$$
G(t, s)= \begin{cases}a_{1} \cos \sqrt{\lambda} t+a_{2} \sin \sqrt{\lambda} t, & 0 \leq t \leq s \leq \eta \\ a_{3} \cos \sqrt{\lambda} t+a_{4} \sin \sqrt{\lambda} t, & s \leq t, s \leq \eta \\ a_{5} \cos \sqrt{\lambda} t+a_{6} \sin \sqrt{\lambda} t, & t \leq s, \eta \leq s \\ a_{7} \cos \sqrt{\lambda} t+a_{8} \sin \sqrt{\lambda} t, & \eta \leq s \leq t \leq 1\end{cases}
$$

The unknown variables $a_{1}, a_{2}, a_{3}$ and $a_{4}$ are found with the help of the definition of Green's function, for any $s \in[0, \eta]$, we have

$$
\begin{aligned}
& a_{1} \cos \sqrt{\lambda} s+a_{2} \sin \sqrt{\lambda} s=a_{3} \cos \sqrt{\lambda} s+a_{4} \sin \sqrt{\lambda} s \\
& \left(-\sqrt{\lambda} a_{1} \sin \sqrt{\lambda} s+a_{2} \sqrt{\lambda} \cos \sqrt{\lambda} s\right)-\left(-\sqrt{\lambda} a_{3} \sin \sqrt{\lambda} s+a_{4} \sqrt{\lambda} \cos \sqrt{\lambda} s\right)=-1,
\end{aligned}
$$

and thus

$$
a_{1}-a_{3}=\frac{1}{\sqrt{\lambda}} \sin \sqrt{\lambda} s, \quad a_{2}-a_{4}=-\frac{1}{\sqrt{\lambda}} \cos \sqrt{\lambda} s .
$$

Then by using the 3 point boundary value condition, we have

$$
a_{1}=0, \quad a_{3} \cos \sqrt{\lambda}+a_{4} \sin \sqrt{\lambda}=\delta\left(a_{3} \cos \sqrt{\lambda} \eta+a_{4} \sin \sqrt{\lambda} \eta\right) .
$$

The values of $a_{1}, a_{2}, a_{3}$ and $a_{4}$ are given by

$$
\begin{aligned}
& a_{1}=0, \quad a_{2}=\frac{\sin \sqrt{\lambda}(1-s)-\delta \sin \sqrt{\lambda}(\eta-s)}{\sqrt{\lambda}(\delta \sin \sqrt{\lambda} \eta-\sin \sqrt{\lambda})}, \\
& a_{3}=-\frac{1}{\sqrt{\lambda}} \sin \sqrt{\lambda} s, \quad a_{4}=-\frac{\sin \sqrt{\lambda} s(\cos \sqrt{\lambda}-\delta \cos \sqrt{\lambda} \eta)}{\sqrt{\lambda}(\delta \sin \sqrt{\lambda} \eta-\sin \sqrt{\lambda})} .
\end{aligned}
$$


Similarly, for any $s \in[\eta, 1]$, we have

$$
\begin{aligned}
& a_{5}=0, \quad a_{6}=\frac{\sin \sqrt{\lambda}(1-s)}{\sqrt{\lambda}(\delta \sin \sqrt{\lambda} \eta-\sin \sqrt{\lambda})}, \\
& a_{7}=-\frac{1}{\sqrt{\lambda}} \sin \sqrt{\lambda} s, \quad a_{8}=\frac{\sin \sqrt{\lambda}(1-s)}{\sqrt{\lambda}(\delta \sin \sqrt{\lambda} \eta-\sin \sqrt{\lambda})}+\frac{1}{\sqrt{\lambda}} \cos \sqrt{\lambda} s .
\end{aligned}
$$

Consequently, we can get the Green's function $G(t, s)$, and lemma is proved.

We can easily prove that the constant sign of Green's function will be nonpositive when $\left(H_{0}\right)$ holds.

Lemma 2. Let $y \in C^{2}(I)$ be a solution of 3 point $B V P(2.1)-(2.2)$, then

$$
y(t)=\frac{b \sin \sqrt{\lambda} t}{\sin \sqrt{\lambda}-\delta \sin \sqrt{\lambda} \eta}-\int_{0}^{1} G(t, s) h(s) d s .
$$

Proof. The 3 point nonhomogeneous linear boundary value problem (2.1)-(2.2) is equivalent to

$$
y(t)=\bar{y}-\int_{0}^{1} G(t, s) h(s) d s,
$$

where $\bar{y}$ is the solution of

$$
L y=0, \quad y(0)=0, \quad y(1)=\delta y(\eta)+b,
$$

and $G(t, s)$ is the solution of

$$
L y=0, \quad y(0)=0, \quad y(1)=\delta y(\eta) .
$$

Suppose

$$
\bar{y}=c_{1} \cos \sqrt{\lambda} t+c_{2} \sin \sqrt{\lambda} t
$$

then by using the boundary value conditions $\bar{y}(0)=(0), \bar{y}(0)=\delta \bar{y}(\eta)+b$, we get

$$
\bar{y}=\frac{b \sin \sqrt{\lambda} t}{\sin \sqrt{\lambda}-\delta \sin \sqrt{\lambda} \eta} .
$$

Hence the boundary value problem $(2.1)-(2.2)$ is equivalent to

$$
y(t)=\frac{b \sin \sqrt{\lambda} t}{\sin \sqrt{\lambda}-\delta \sin \sqrt{\lambda} \eta}-\int_{0}^{1} G(t, s) h(s) d s .
$$

Case II: $\lambda<0$. Let us assume

$\left(H_{0}^{\prime}\right) \quad \lambda<0, \cosh \sqrt{|\lambda|}-\delta \cosh \sqrt{|\lambda|} \eta \geq 0$ and $\delta \sinh \sqrt{|\lambda|} \eta-\sinh \sqrt{|\lambda|}<0$.

We can easily verify that, there is a range of $\lambda<0$, which support $\left(H_{0}^{\prime}\right)$ (see Figure 6). 
Lemma 3. The Green's function of the 3 point $B V P L y=0, y(0)=0, y(1)=$ $\delta y(\eta)$ for $\lambda<0$ is

$$
G(t, s)=k_{2} \begin{cases}{[\sinh \sqrt{|\lambda|}(1-s)-\delta \sinh \sqrt{|\lambda|}(\eta-s)] \sinh \sqrt{|\lambda|} t,} & 0 \leq t \leq s \leq \eta, \\ \sinh \sqrt{|\lambda|} s[\sinh \sqrt{|\lambda|}(1-t)-\delta \sinh \sqrt{|\lambda|}(\eta-t)], & s \leq t, s \leq \eta, \\ \sinh \sqrt{|\lambda|}(1-s) \sinh \sqrt{|\lambda|} t, & t \leq s, \eta \leq s, \\ \delta \sinh \sqrt{|\lambda|} \eta \sinh \sqrt{|\lambda|}(t-s) & \\ \quad+\sinh \sqrt{|\lambda|} s \sinh \sqrt{|\lambda|}(1-t), & \eta \leq s \leq t \leq 1,\end{cases}
$$

where $k_{2}=\frac{1}{\sqrt{|\lambda|}(\delta \sinh \sqrt{|\lambda|} \eta-\sinh \sqrt{|\lambda|})}$. If $\left(H_{0}^{\prime}\right)$ is true then $G(t, s) \leq 0$.

Lemma 4. Let $y \in C^{2}(I)$ be a solution of 3 point $B V P(2.1)-(2.2)$. Then $y(t)$ is given by the following equation

$$
y(t)=\frac{b \sinh \sqrt{|\lambda|} t}{\sinh \sqrt{|\lambda|}-\delta \sinh \sqrt{|\lambda|} \eta}-\int_{0}^{1} G(t, s) h(s) d s .
$$

\subsection{Existence of some differential inequalities}

In this section we prove existence of some differential inequalities which govern the range of $\lambda$ and also they ensure that if these inequalities are true the solutions generated by iterative scheme are monotonic.

Lemma 5. Let $M \in R^{+}$and $N:[0,1] \rightarrow[0, \infty)$ such that $N(0)=0, N^{\prime}(t) \geq 0$. If $0<\lambda<\frac{\Pi^{2}}{4}$ is such that $\lambda-M \leq 0$ and

(i) if $(\lambda-M) \cos \sqrt{\lambda}+N(t) \sqrt{\lambda} \sin \sqrt{\lambda} \leq 0$, then for all $t \in[0,1]$

$$
(\lambda-M) \cos \sqrt{\lambda} t+N(t) \sqrt{\lambda} \sin \sqrt{\lambda} t \leq 0 .
$$

(ii) If $\lambda+\sup N^{\prime}(t) \leq M$, then for all $t \in[0,1]$

$$
(\lambda-M) \sin \sqrt{\lambda} t+N(t) \sqrt{\lambda} \cos \sqrt{\lambda} t \leq 0 .
$$

Proof. The function

$$
(\lambda-M) \cos \sqrt{\lambda} t+N(t) \sqrt{\lambda} \sin \sqrt{\lambda} t
$$

is non-decreasing for all $t \in[0,1]$ and satisfy the following inequality,

$$
(\lambda-M) \cos \sqrt{\lambda} t+N(t) \sqrt{\lambda} \sin \sqrt{\lambda} t \leq(\lambda-M) \cos \sqrt{\lambda}+N(t) \sqrt{\lambda} \sin \sqrt{\lambda} .
$$

By using the assumptions it is easy to verify (i).

Using the properties of sin, cos and assumptions, we can easily see that for all $t \in[0,1]$,

$$
(\lambda-M) \sin \sqrt{\lambda} t+N(t) \sqrt{\lambda} \cos \sqrt{\lambda} t \leq 0 .
$$

Hence (ii) is verified. 
Lemma 6. Let $M \in R^{+}$and $N:[0,1] \rightarrow[0, \infty)$ such that $N(0)=0$. If $\lambda<0$ is such that $M+\lambda \leq 0$, and

(i) if $[(M+\lambda)+N \sqrt{|\lambda|}] \leq 0$, then for all $t \in[0,1]$

$$
(M+\lambda) \cosh \sqrt{|\lambda|} t+N(t) \sqrt{|\lambda|} \sinh \sqrt{|\lambda|} t \leq 0 .
$$

(ii) If $(M+\lambda)+N^{\prime}(t)+N(t) \sqrt{|\lambda|} \leq 0$, then for all $t \in[0,1]$

$$
(M+\lambda) \sinh \sqrt{|\lambda|} t+N(t) \sqrt{|\lambda|} \cosh \sqrt{|\lambda|} t \leq 0 .
$$

Proof. As

$$
(M+\lambda) \cosh \sqrt{|\lambda|} t+N(t) \sqrt{|\lambda|} \sinh \sqrt{|\lambda|} t \leq[(M+\lambda)+N(t) \sqrt{|\lambda|}] \cosh \sqrt{|\lambda|} t .
$$

The right hand side of the above inequality will be non-positive for all $t \in[0,1]$, if

$$
[(M+\lambda)+N(t) \sqrt{|\lambda|}] \leq 0 .
$$

This complete the part (i) of the lemma.

Using the assumptions and the properties of sinh and cosh, we can easily see that for all $t \in[0,1]$

$$
(M+\lambda) \sinh \sqrt{|\lambda|} t+N(t) \sqrt{|\lambda|} \cosh \sqrt{|\lambda|} t
$$

is a non-increasing function, which proves part (ii).

Lemma 7. Let $\left(H_{0}\right)$ be true. If $y(t)$ is the solution of $(2.1)-(2.2)$ then we have

(i) $G(t, s) \leq 0$ and

(ii) $(\lambda-M) G(t, s)+N(t)\left(\operatorname{sign} y^{\prime}\right) \frac{\partial G(t, s)}{\partial t} \geq 0$

for any $t, s \in[0,1]$ and $t \neq s$.

Proof. The condition $\left(H_{0}\right)$ guarantees that $G(t, s) \leq 0$. Putting the value of $G(t, s)$ and $\frac{\partial G(t, s)}{\partial t}$ for $t \neq s$ in $(\lambda-M) G(t, s)+N(t)\left(\operatorname{sign} y^{\prime}\right) \frac{\partial G(t, s)}{\partial t}$, and using the Lemma 5 , we can prove that

$$
(\lambda-M) G(t, s)+N(t)\left(\operatorname{sign} y^{\prime}\right) \frac{\partial G(t, s)}{\partial t} \geq 0 .
$$

Lemma 8. Let $\left(H_{0}^{\prime}\right)$ be true. Let $y(t)$ be the solution of $(2.1)-(2.2)$ then we have

(i) $G(t, s) \leq 0$, for any $t, s \in[0,1]$,

(ii) $(M+\lambda) G(t, s)+N(t)\left(\operatorname{sign} y^{\prime}\right) \frac{\partial G(t, s)}{\partial t} \geq 0$

for any $t, s \in[0,1]$ and $t \neq s$.

Proof. By Lemma 6 and Lemma 7 we arrive at Lemma 8. 


\subsection{Maximum principle}

We conclude the following two results.

Proposition 1. Suppose that $\left(H_{0}\right)$ holds. Let $y(t)$ be the solution of (2.1)(2.2) and if $b \geq 0, h(t) \in C[0,1]$ be such that $h(t) \geq 0$, then $y(t) \geq 0$.

Proposition 2. Suppose that $\left(H_{0}^{\prime}\right)$ holds. Let $y(t)$ be the solution of (2.1)(2.2) and if $b \geq 0, h(t) \in C[0,1]$ be such that $h(t) \geq 0$, then $y(t) \geq 0$.

\section{Nonlinear 3 Point BVP}

In this section we consider the nonlinear 3 point BVP. We show that it is possible to find out a range of $\lambda \neq 0$ on $\lambda$ axis so that the iterative scheme (1.3)-(1.4) generates monotone sequences. Which finally proves existence of nonlinear 3 point BVP (1.1)-(1.2).

We define lower solution and upper solution represented by functions $\alpha(t)$ and $\beta(t)$, respectively, such that $\alpha \leq \beta$.

Definition 1 . Let $\alpha, \beta \in C^{2}[0,1]$. Then $\alpha(t)$ and $\beta(t)$ are called lower solution and upper solution of the nonlinear 3 point BVP (1.1)-(1.2) if they satisfy

$$
\begin{aligned}
& -\alpha^{\prime \prime}(t) \leq f\left(t, \alpha, \alpha^{\prime}\right), \quad 0<t<1, \\
& \alpha(0)=0, \quad \alpha(1) \leq \delta \alpha(\eta)
\end{aligned}
$$

and

$$
\begin{aligned}
& -\beta^{\prime \prime}(t) \geq f\left(t, \beta, \beta^{\prime}\right), \quad 0<t<1, \\
& \beta(0)=0, \quad \beta(1) \geq \delta \beta(\eta) .
\end{aligned}
$$

Our proof is based on uniform convergence of the sequences and for that we use Arzela-Ascoli theorem. To implement this we need equicontinuity and equiboundedness of $\left\{y_{n}\right\}$ and $\left\{y_{n}^{\prime}\right\}$. Equicontinuity and equiboundedness of $y_{n}$ and $y_{n}^{\prime}$ can be proved by continuity of the Green's function and continuity of the solution on $[0,1]$ and continuity of the nonlinear term $f\left(t, y, y^{\prime}\right)$. Equiboundedness of $\left\{y_{n}^{\prime}\right\}$ is established by the following two lemmas.

\subsection{Priori bound}

$\left(H_{P}\right)$ Let $|f(t, u, v)| \leq \varphi(|v|)$ for all $(t, u, v) \in D$. Assume that

$$
\varphi: R^{+} \rightarrow R^{+}
$$

is continuous and satisfies $\max _{t \in[0,1]} \beta-\min _{t \in[0,1]} \alpha \leq \int_{l_{0}}^{\infty} \frac{s d s}{\varphi(s)}$. Here $l_{0}=\sup _{[0,1]}|\beta(t)|$.

Lemma 9. Assume that $f\left(t, y, y^{\prime}\right)$ satisfies $\left(H_{P}\right)$. Then there exists $R>0$ such that any solution of

$$
\begin{aligned}
& -y^{\prime \prime}(t) \geq f\left(t, y, y^{\prime}\right), \quad 0<t<1, \\
& y(0)=0, \quad y(1) \geq \delta y(\eta)
\end{aligned}
$$

with $y \in[\alpha(t), \beta(t)]$ for all $t \in[0,1]$ satisfies $\left\|y^{\prime}\right\|_{\infty} \leq R$. 
Proof. The proof can be divided in two parts.

Case $(i)$. If solution is not monotone in $[0,1]$, then consider the interval $\left(t_{0}, t\right] \subset(0,1)$ such that $y^{\prime}\left(t_{0}\right)=0$ and $y^{\prime}(t)>0$ for $t>t_{0}$. Integrating (3.1) from $t_{0}$ to $t$ we get

$$
\int_{0}^{y^{\prime}} \frac{s d s}{\varphi(s)} \leq \max _{t \in[0,1]} \beta-\min _{t \in[0,1]} \alpha .
$$

From $\left(H_{P}\right)$ we can choose $R>0$ such that

$$
\int_{0}^{y^{\prime}} \frac{s d s}{\varphi(s)} \leq \max _{t \in[0,1]} \beta-\min _{t \in[0,1]} \alpha \leq \int_{l_{0}}^{R} \frac{s d s}{\varphi(s)} \leq \int_{0}^{R} \frac{s d s}{\varphi(s)},
$$

which gives

$$
y^{\prime}(t) \leq R
$$

Now we consider the case in which $y^{\prime}(t)<0$ for $t<t_{0}, y^{\prime}\left(t_{0}\right)=0$, and proceeding in the similar way we get

$$
-y^{\prime}(t) \leq R
$$

and the result follows.

Case $(i i)$. If $y$ is monotonically decreasing in $(0,1)$, that is $y^{\prime}(t)<0$ in $t \in(0,1]$ then by mean value theorem there exists a point $\tau \in(0,1)$ such that

$$
-y^{\prime}(\tau) \leq|\beta| .
$$

Now, integrating (3.1) from $t$ to $\tau$, using $\left(H_{P}\right)$ we can choose $R$, such that $-y^{\prime} \leq R$.

Similarly if $y$ is monotonically increasing in $(0,1)$, that is $y^{\prime}(t)>0$ in $t \in(0,1]$ proof can be completed as above.

Lemma 10. If $f\left(t, y, y^{\prime}\right)$ satisfies $\left(H_{P}\right)$, then there exists $R>0$ such that any solution of

$$
\begin{aligned}
& -y^{\prime \prime}(t) \leq f\left(t, y, y^{\prime}\right), \quad 0<t<1, \\
& y(0)=0, \quad y(1) \leq \delta y(\eta)
\end{aligned}
$$

with $y \in[\alpha(t), \beta(t)]$ for all $t \in[0,1]$ satisfies $\left\|y^{\prime}\right\|_{\infty} \leq R$.

Proof. Proof follows from the analysis of Lemma 9.

Now we state the existence Theorem 1 (for $\lambda>0$ ) and Theorem 2 (for $\lambda<0)$ which are the main results of our paper.

Theorem 1. Let $\left(H_{0}\right)$ be true. Further assume that

$\left(H_{1}\right)$ there exist $\alpha$ and $\beta \in C^{2}[0,1]$, lower and upper solutions of (1.1)-(1.2) such that for all $t \in[0,1], \alpha \leq \beta$; 
$\left(H_{2}\right)$ the function $f: D \rightarrow R$ is continuous on $D:=\left\{(t, u, v) \in[0,1] \times R^{2}\right.$ : $\alpha(t) \leq u \leq \beta(t)\}$

$\left(H_{3}\right)$ there exist $M \geq 0$ such that for all $\left(t, u_{1}, v\right),\left(t, u_{2}, v\right) \in D$

$$
u_{1} \leq u_{2} \Longrightarrow f\left(t, u_{2}, v\right)-f\left(t, u_{1}, v\right) \geq M\left(u_{2}-u_{1}\right)
$$

$\left(H_{4}\right)$ there exist $N:[0,1] \rightarrow[0, \infty)$ such that $N(0)=0, N^{\prime}(t) \geq 0$ and for all $\left(t, u, v_{1}\right),\left(t, u, v_{2}\right) \in D$

$$
\left|f\left(t, u, v_{2}\right)-f\left(t, u, v_{1}\right)\right| \leq N(t)\left|v_{2}-v_{1}\right| .
$$

$\left(H_{5}\right)$ Let $\lambda>0$ be such that $\lambda-M \leq 0,(\lambda-M) \cos \sqrt{\lambda}+N(t) \sqrt{\lambda} \sin \sqrt{\lambda} \leq 0$ and $\lambda+\sup N^{\prime}(t) \leq M$, and for all $t \in[0,1]$

$$
f\left(t, \beta(t), \beta^{\prime}(t)\right)-f\left(t, \alpha(t), \alpha^{\prime}(t)\right)-\lambda(\beta-\alpha) \geq 0,
$$

then the sequences $\left(\alpha_{n}\right)$ and $\left(\beta_{n}\right)$ defined by (1.3)-(1.4) converges monotonically in $C^{1}([0,1])$ to solution $v$ and $u$ of $(1.1)-(1.2)$, such that for all $t \in[0,1]$

$$
\alpha \leq v \leq u \leq \beta
$$

Proof. With the help of assumptions $\left(H_{1}\right),\left(H_{2}\right),\left(H_{3}\right),\left(H_{4}\right)$ and $\left(H_{5}\right)$, we conclude that

$$
\alpha=\alpha_{0} \leq \alpha_{1} \leq \alpha_{2} \leq \cdots \leq \alpha_{n} \leq \cdots \leq \beta_{n} \leq \cdots \leq \beta_{2} \leq \beta_{1} \leq \beta_{0}=\beta .
$$

It is clear that the sequences $\left(\alpha_{n}\right)_{n}$ and $\left(\beta_{n}\right)_{n}$ are monotonic and bounded. Hence they converge to the functions $v(t)$ and $u(t)$ (say), respectively, which are such that for all $n, \alpha_{n} \leq v \leq u \leq \beta_{n}$.

By using the equations (1.3)-(1.4), inequality (3.2) and Lemma 9, 10, we prove that the sequences $\left(\beta_{n}\right)_{n}$ and $\left(\alpha_{n}\right)_{n}$ are equibounded and equicontinuous in $C^{1}([0,1])$, i.e., any subsequence of $\left(\beta_{n}\right)_{n}$ and $\left(\alpha_{n}\right)_{n}$ are also equibounded and equicontinuous in $C^{1}([0,1])$. Now by using Arzela-Ascoli theorem, we conclude that the subsequences of $\left(\beta_{n}\right)_{n}$ and $\left(\alpha_{n}\right)_{n}$ contain a subsequence which converge uniformly in $C^{1}([0,1])$.

By uniqueness of the limit and monotonicity of the sequences $\left(\alpha_{n}\right)_{n}$ and $\left(\beta_{n}\right)_{n}$, we have $\alpha_{n} \rightarrow v$ and $\beta_{n} \rightarrow u$.

We write the solution of iterative scheme (1.3)-(1.4) for both $\left(\alpha_{n}\right)$ and $\left(\beta_{n}\right)$ by using Lemma 2 , where $h(t)$ is in terms of nonlinear term $f$. Now by using uniform convergence, one can easily conclude the existence of the solution of nonlinear 3 point BVP. This completes the proof.

Theorem 2. Let $\left(H_{0}^{\prime}\right),\left(H_{1}\right),\left(H_{2}\right)$ and $\left(H_{4}\right)$ be true. Further assume that $\left(H_{1}^{\prime}\right)$ there exist $M \geq 0$ such that for all $\left(t, u_{1}, v\right),\left(t, u_{2}, v\right) \in D$

$$
u_{1} \leq u_{2} \Longrightarrow f\left(t, u_{2}, v\right)-f\left(t, u_{1}, v\right) \geq-M\left(u_{2}-u_{1}\right) .
$$


$\left(H_{2}^{\prime}\right)$ Let $\lambda<0$ be such that $M+\lambda \leq 0,(M+\lambda)+N^{\prime}(t)+N(t) \sqrt{|\lambda|} \leq 0$, $[(M+\lambda)+N \sqrt{|\lambda|}] \leq 0$ and for all $t \in[0,1]$

$$
f\left(t, \beta(t), \beta^{\prime}(t)\right)-f\left(t, \alpha(t), \alpha^{\prime}(t)\right)-\lambda(\beta-\alpha) \geq 0,
$$

then the sequences $\left(\alpha_{n}\right)$ and $\left(\beta_{n}\right)$ defined by (1.3)-(1.4) converges monotonically in $C^{1}([0,1])$ to solution $v$ and $u$ of $(1.1)-(1.2)$, such that for all $t \in[0,1], \alpha \leq v \leq u \leq \beta$.

Proof. Proof is same as Theorem 1.

\section{Numerical Illustrations}

To verify our results, we consider two examples for both $\lambda>0, \lambda<0$ and show that it is possible to compute a range of $\lambda$ so that iterative scheme generates monotone sequences which converge to the solution of nonlinear problem.

Example 1. Consider the following 3 point BVP

$$
\begin{aligned}
& -y^{\prime \prime}(t)=\left(\frac{9 e^{y}+t e^{y^{\prime}}}{15}\right), \quad 0<t<1, \\
& y(0)=0, \quad y(1)=0.95 y(0.2) .
\end{aligned}
$$

This problem has $\alpha=0$ and $\beta=3\left(t-\frac{t^{2}}{2}\right)$ as lower and upper solutions. The nonlinear term is Lipschitz in both $y$ and $y^{\prime}$ and continuous for all values of $y$ and $y^{\prime}$. It is easy to see that Nagumo condition is given by

$$
|f(t, u, v)| \leq \frac{9}{15} e^{\frac{3}{2}}+\frac{1}{15} e^{v}
$$

i.e., $\varphi=\frac{9}{15} e^{\frac{3}{2}}+\frac{1}{15} e^{v}$. Using Lemma 9 we can compute easily that $\left|y^{\prime}\right| \leq \sqrt{\frac{1}{10}}$, i.e., $R=0.316228$. The Lipschitz constants are $M=\frac{3}{5}$ and $N(t)=\frac{t}{15} e^{R}$. In Figures $1-5$ we describe monotonic behavior or some inequalities and solutions. In Figure 1 we have verified that for it is possible to get a range of $\lambda$ such that $\left(H_{0}\right)$ is true. Then from Figure 2 to Figure 5 we have shown that for different values of $\lambda \in[0.15,0.49]$ monotonic sequences are obtained and both converge to solutions of Example 1. In this range all the inequalities are also true which are required to generate monotonic sequences. The range $[0.15,0.49]$ is not sharp and is based on computations done in Mathematica 7.0. In Figure 11 we have shown that if $\lambda$ is not in the range $[0.15,0.49]$ then monotonicity is lost.

Example 2. Consider the boundary value problem

$$
\begin{aligned}
& -y^{\prime \prime}(t)=\frac{\left(e^{t}-1\right)}{36}\left[\left(y^{\prime}(t)\right)^{2}-y(t)-\frac{\cos t}{4}\right], \quad 0<t<1, \\
& y(0)=0, \quad y(1)=0.5 y(0.5)
\end{aligned}
$$

Here $f\left(t, y, y^{\prime}\right)=\frac{\left(e^{t}-1\right)}{36}\left[\left(y^{\prime}(t)\right)^{2}-y(t)-\frac{\cos t}{4}\right], \delta=0.5, \eta=0.5$. This problem has $\alpha=\left(\frac{t^{2}}{4}-t\right)$ and $\beta=\frac{t}{2}$ as lower and upper solutions. The nonlinear term 
is Lipschitz in both $y$ and $y^{\prime}$ and continuous for all values of $y$ and $y^{\prime}$. It is easy to see that Nagumo condition is given by

$$
|f(t, u, v)| \leq 0.0477301\left(|v|^{2}+1\right),
$$

i.e., $\varphi=0.0477301\left(|v|^{2}+1\right)$. Using Lemma 10 we can compute bound for $y^{\prime}$, i.e., $\left|y^{\prime}\right| \leq \frac{1}{2} e^{\frac{5}{4}(0.0477301)}$, i.e., $R=0.530739$. The Lipschitz constants are $M=0.0477301$ and $N(t)=\frac{\left(e^{t}-1\right)}{36}(1.06148)$.

In Figure 6 we have verified that for $\lambda<-1,\left(H_{0}^{\prime}\right)$ is true. In Figures 7-10 we describe monotonic behavior of the sequences. For $\lambda<-1$ all the inequalities required are also valid. The upper bound for $\lambda$ is not sharp and is based on computations done on Mathematica 7.0. Then from Figure 7 to Figure 10 we have shown that for different values of $\lambda$ monotonic sequences are obtained and both converge to solutions of Example 2. Here it is also visible from the Figure 10 that sequence thus obtained are uniformly convergent.

\section{Conclusion}

In this paper we have considered an iterative scheme which is simple enough for computational point of view. We did not consider $\lambda$ as function of $t$. The method developed in this paper can be coded to generate a user friendly package which can be efficiently used to compute solutions of the nonlinear 3 point BVP whose close form solutions is not known.

We have constructed two examples one for each case $\lambda>0$ and $\lambda<0$ and show that derived sufficient conditions can generate solutions for a class of nonlinear 3 point BVPs. Mainly it is initial iterates (upper and lower solutions) choice of which matters and success of the method depends on them. If initial iterates are chosen properly then it is guaranteed that sequences will converge to the solutions of the nonlinear BVP. In Figure 11 we also observe that if $\lambda$ does not belong to the range sequences are not monotone.

We also observe that Remark 5.4 of Cherpion et al. [2] seems to be true even in case of 3 point BVP with Dirichlet type boundary condition.

\section{Acknowledgement}

We would like to thank anonymous reviewers for their expert comments which helped us a lot, and paper is improved. This work is partially supported by Grant provided by UGC, New Delhi, India, File No. F.4-1/2006 (BSR)/7-203/ 2009(BSR). 


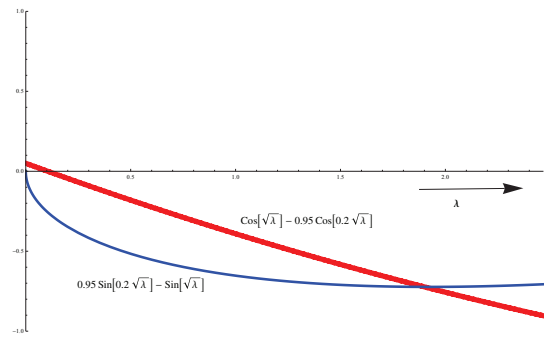

Figure 1. $\left(H_{0}\right)$.

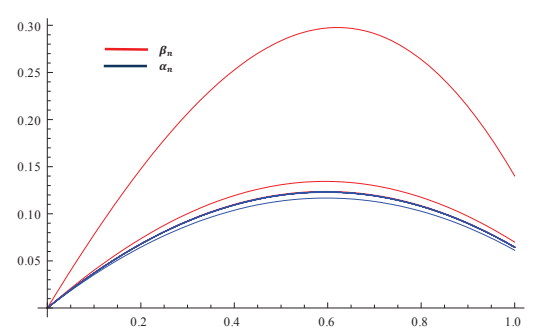

Figure 3. $\lambda=0.3$ and $n=1,2, \ldots, 10$.

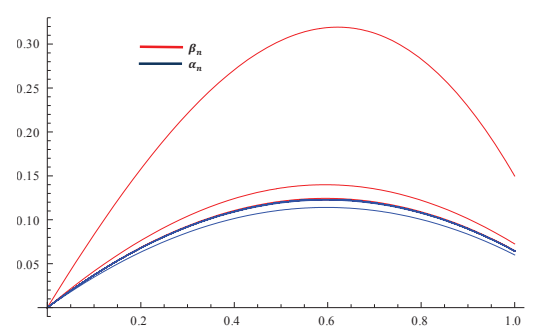

Figure 5. $\lambda=0.15$ and $n=1,2, \ldots, 30$.

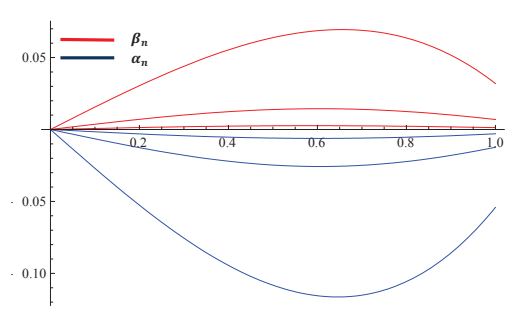

Figure 7. $\lambda=-2$ and $n=1,2,3$.

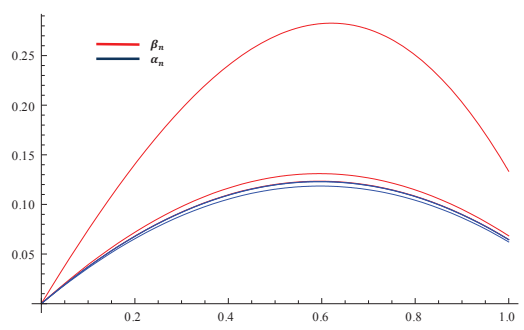

Figure 2. $\lambda=0.4$ and $n=1,2,3$.

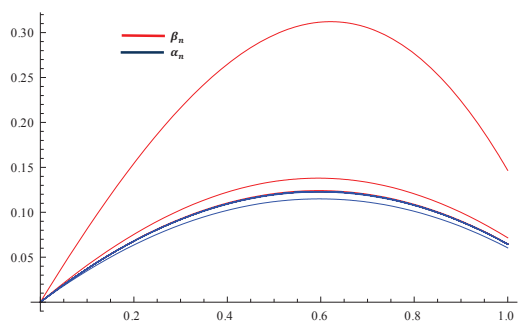

Figure 4. $\lambda=0.2$ and $n=1,2, \ldots, 20$.

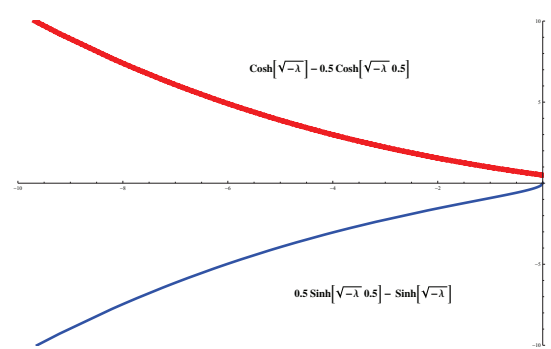

Figure 6. $\left(H_{0}^{\prime}\right)$.

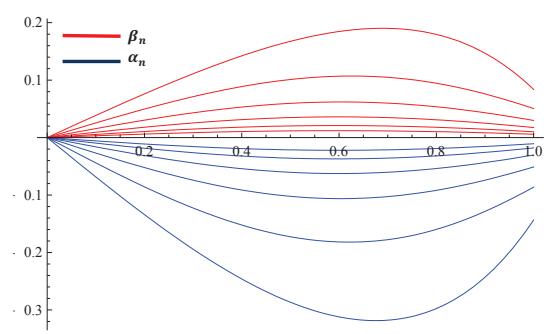

Figure 8. $\lambda=-10$ and $n=1,2, \ldots, 6$. 

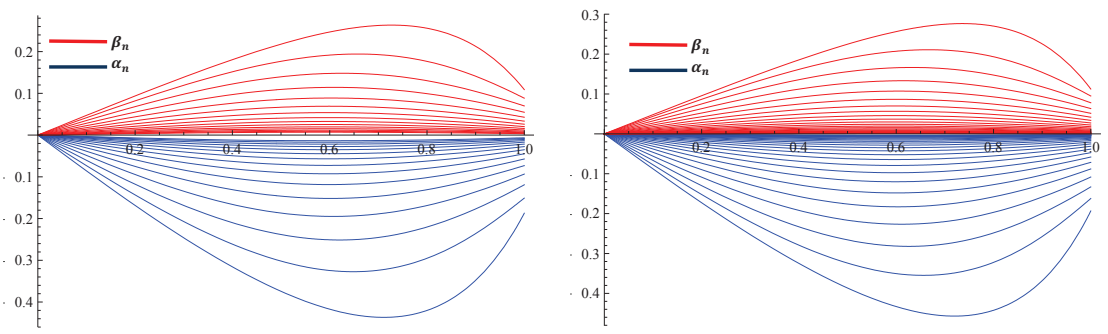

Figure 9. $\lambda=-25$ and $n=1,2, \ldots, 15$.

Figure 10. $\lambda=-30$ and $n=1,2, \ldots, 25$.

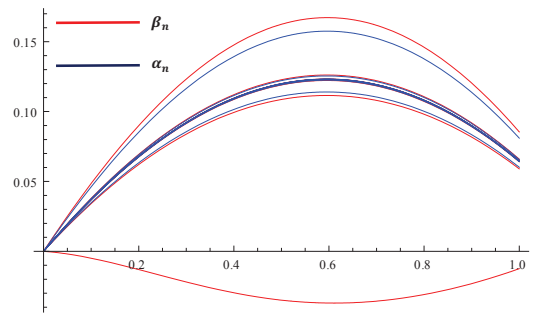

Figure 11. Non-monotonicity for $\lambda=2$.

\section{References}

[1] G. Bao, X. Xu and Y. Song. Positive solutions for three-point boundary value problems with a non-well-ordered upper and lower solution condition. Appl. Math. Lett., 25:767-770, 2012. http://dx.doi.org/10.1016/j.aml.2011.10.017.

[2] M. Cherpion, C. De Coster and P. Habets. A constructive monotone iterative method for second-order BVP in the presence of lower and upper solutions. Appl. Math. Comput., 123:75-91, 2001.

http://dx.doi.org/10.1016/S0096-3003(00)00058-8.

[3] I. Kiguradze and B. Puza. On boundary value problems for functional-differential equations. Mem. Differential Equations Math. Phys., 12:106-113, 1997.

[4] I.T. Kiguradze and A.G. Lomtatidze. On certain boundary value problems for second-order linear ordinary differential equations with singularities. J. Math. Anal. Appl., 101:325-347, 1984.

http://dx.doi.org/10.1016/0022-247X(84)90107-0.

[5] F. Li, M. Jia, X. Liu, C. Li and G. Li. Existence and uniqueness of solutions of second-order three-point boundary value problems with upper and lower solutions in the reversed order. Nonlinear Anal., 68:2381-2388, 2008. http://dx.doi.org/10.1016/j.na.2007.01.065.

[6] F. Li, J. Sun and M. Jia. Monotone iterative method for the second-order threepoint boundary value problem with upper and lower solutions in the reversed order. Appl. Math. Comput., 217:4840-4847, 2011.

http://dx.doi.org/10.1016/j.amc.2010.11.003. 
[7] A. Lomtatidze and P. Vodstrch. On sign constant solutions of certain boundary value problems for second-order functional differential equations. Appl. Anal., 84:197-209, 2005. http://dx.doi.org/10.1080/00036810410001724427.

[8] A.G. Lomtatidze. A boundary value problem for nonlinear second order ordinary differential equations with singularities. Differ. Uravn., 22:416-426, 1986.

[9] A.G. Lomtatidze. Positive solutions of boundary value problems for second-order ordinary differential equations with singularities. Differ. Uravn., 23:1685-1692, 1987.

[10] A.G. Lomtatidze. A nonlocal boundary value problem for second-order linear ordinary differential equations. Differ. Uravn., 31:446-455, 1995.

[11] A.G. Lomtatidze. On a nonlocal boundary value problem for second-order linear ordinary differential equations. J. Math. Anal. Appl., 193:889-908, 1995. http://dx.doi.org/10.1006/jmaa.1995.1273.

[12] A.G. Lomtatidze and L. Malaguti. On a nonlocal boundary value problem for second-order nonlinear singular differential equations. Georgian Math. J., 7:133$154,2000$.

[13] S. Roman and A. Štikonas. Green's functions for stationary problems with four-point nonlocal boundary conditions. In V. Kleiza, S. Rutkauskas and A. Štikonas(Eds.), Proceedings of International Conference, Differential Equations and Their Applications (DETA-2009), September 10-12, 2009, Panevežys, Lithuania, pp. 123-130, Kaunas University of Technology, 2009.

[14] S. Roman and A. Štikonas. Green's functions for stationary problems with nonlocal boundary conditions. Lith. Math. J., 49(2):190-202, 2009. http://dx.doi.org/10.1007/s10986-009-9041-0.

[15] M. Singh and A.K. Verma. On a monotone iterative method for a class of three point nonlinear nonsingular bvps with upper and lower solutions in reverse order. J. Appl. Math. Comput., 43:99-114, 2013. http://dx.doi.org/10.1007/s12190-013-0654-4.

[16] M. Singh and A.K. Verma. Picard type iterative scheme with initial iterates in reverse order for a class of nonlinear three point bvps. J. Appl. Math. Comput., 2013:Article ID 728149, 6 pages, 2013.

[17] A. Štikonas. A survey on stationary problems, Green's functions and spectrum of Sturm-Liouville problem with nonlocal boundary conditions. Nonlinear Anal. Model. Control, 19(3):301-334, 2014. http://dx.doi.org/10.15388/NA.2014.3.1.

[18] A. Štikonas and S. Roman. Stationary problems with two additional conditions and formulae for Green's functions. Numer. Funct. Anal. Optim., 30(9):11251144, 2009. http://dx.doi.org/10.1080/01630560903420932.

[19] J.R.L. Webb. Existence of positive solutions for a thermostat model. Nonlinear Anal. Real World Appl., 13:923-938, 2012.

http://dx.doi.org/10.1016/j.nonrwa.2011.08.027.

[20] Z. Wei and C. Pang. The method of lower and upper solutions for fourth order singular $m$-point boundary value problems. J. Math. Anal. Appl., 322:675-692, 2006. http://dx.doi.org/10.1016/j.jmaa.2005.09.064.

[21] Y. Zou, Q. Hu and R. Zhang. On numerical studies of multi-point boundary value problem and its fold bifurcation. Appl. Math. Comput., 185:527-537, 2007. http://dx.doi.org/10.1016/j.amc.2006.07.064. 